\title{
The Effect of Chemoprophylaxis for Exposed Individuals on Lymphatic Filariasis Model
}

\author{
Aziza Juma Iddi ${ }^{1, ~}$, Estomih Massawe ${ }^{1}$, Gamba Nkwengulila ${ }^{2}$, Moatlhodi Kgosimore ${ }^{3}$ \\ ${ }^{1}$ Mathematics Department, University of Dar Es Salaam, Dar Es Salaam, Tanzania \\ ${ }^{2}$ Zoology and Wildlife Conservation Department, University of Dar Es Salaam, Dar Es Salaam, Tanzania \\ ${ }^{3}$ Mathematics Department, Botswana College of Agriculture, Gaborone, Botswana
}

Email address:

iddiaziza@gmail.com (A. J. Iddi), estomihmassawe@yahoo.com (E. Massawe), gamba@udsm.ac.tz (G. Nkwengulila), kgosimor@gmail.com (M. Kgosimore),mkgosi@bca.bw (M. Kgosimore)

\section{To cite this article:}

Aziza Juma Iddi, Estomih Massawe, Gamba Nkwengulila, Moatlhodi Kgosimore. The Effect of Chemoprophylaxis for Exposed Individuals on Lymphatic Filariasis Model. Applied and Computational Mathematics. Vol. 5, No. 1, 2016, pp. 30-39. doi: 10.11648/j.acm.20160501.15

\begin{abstract}
In this paper, a deterministic Lymphatic Filariasis (LF) model is formulated and analyzed with the aim of assessing the effect of chemoprophylaxis for the exposed individuals and treatment of symptomatic LF infections. Qualitative and quantitative analysis are implemented to determine the basic reproduction number $R_{e}$ necessary for the control of the diseases in the communities. The disease-free equilibrium (DFE) exists and is locally and globally asymptotically stable if $R_{e}<1$, whereas if $R_{e}>1$ the endemic equilibrium exists and it is locally asymptotically stable. Numerical simulations are carried to complement the analytical results.
\end{abstract}

Keywords: Chemoprophylaxis, Exposed Individuals, Lymphatic Filariasis

\section{Introduction}

Lymphatic Filariasis (LF), a debilitating disease, is one of the most prevalent and yet the most neglected tropical diseases with serious economic and social consequences Bhunu [1]. Lymphatic Filariasis is more common in regions with high incidence of poverty, making it a disease of the poor and serves as an indicator of underdevelopment Tan [21].

Chemoprophylaxis or Chemoprevention refers to the administration of a medication for the purpose of preventing or curing the disease or infection in the community. The Lymphatic Filariasis disease has three broad clinical scenarios which are asymptomatic infection, acute infection and chronic infection.

Chemoprophylaxis being one of the approaches to prevent LF includes giving medicine that kills the microscopic worms and suppress the level of microfilariae in the blood and thereby reduce the transmission of parasites by mosquitoes in endemic areas. Chan [4] observed that LF disease progression is a consequence of worm induced damage and occurs at a high rate of hydrocele and low rate of lymphedema.

The present strategies to control LF disease include the use of Mass Drug Administration (MDA) of which the whole community are given anti-filarial drugs irrespective of their mf-status. A number of studies on the impact of MDA on the transmission dynamics of LF infections have been developed over the years (decades). For instance, Stolk [18] developed LYMFASIM, a microsimulation model for transmission and control of lymphatic filariasis, the authors used the model to simulate the effects of mass treatment, in order to estimate the number of treatment rounds necessary to achieve elimination of the disease. The study revealed that, the effectiveness of mass treatment depend much on number of treatment rounds, coverage, drug efficacy and endemicity level.

Stolk [17] compared EPIFIL and LYMFASIM models in terms of their structure and parameter quantifications and highlighted deficiencies that impede their wide spread application for decision support. They found that, despite differences in model structure and parameterization, both models were able to predict the duration of control through treatment required for elimination under assumptions based on biological understanding.

Jambulingam [8]. Used LYMFASIM model to estimate the duration of MDA required for elimination and residual levels 
of microfilaemia (Mf) and antigenaemia (Ag) prevalence reached after that duration in different transmission settings varying from low to high. The result indicated that the duration of annual MDA increased with higher baseline endemicity and lower coverage.

With the 2020 goal of elimination looming, Kastner [9] used a model-based assessment to develop plausible scale-up scenarios to reach global elimination and eradication of LF.

Authors predicted the duration of MDA to reach local elimination for different transmission archetypes, estimated the required number of treatments and the implication of rapid scale-up. The result showed that, if MDA programs are drastically scaled up and expanded, the final round of MDA for LF eradication could be delivered in 2028 after 4,159 million treatments.

Swaminathan [19] developed two mathematical models EPIFIL and LYMFASIM. Both models have been used to predict the long-term impact of control programmes (mass chemotherapy/vector control) and assess the prospects of elimination. They suggested that, although progression to hydrocoele may be associated with worm burden, progression to lymphoedema is due to immunopathological reactions and secondary bacterial infections. Further progression of lymphedema depends on the frequency of acute episodes of adenolymphangitis.

Bhunu [1] developed an analytical deterministic model for the spread of lymphatic Filariasis, a mosquito-borne infection. The author found that even when all lymphatic filariasis cases displaying elephantiasis symptoms are put on treatment it will not be able to eradicate the disease. The result suggests that effective control of lymphatic filariasis may lie in treatment for those displaying symptoms as well as chemoprophylaxis for the exposed.

This work differs from others by introducing treatment at early stage of infection (asymptomatic) for the purpose of reducing the number of infected individuals, as also suggested by Bhunu [1].

Therefore in this study, we propose and analyse an analytical deterministic model of the transmission dynamics of lymphatic Filariasis with the aim of obtaining insight on the effect of chemoprophylaxis for the exposed class and treatment of asymptomatic LF infections in a population.

\section{Model Formulation}

We formulate a Lymphatic Filariasis model in which the human population is divided into four (4) compartments and the vector population is divided into three (3) compartments distinguished by disease status. The human population at time $t$, $N_{h}(t)$ comprise the susceptible compartment $S_{h}(t)$ made of individuals who are at risk of LF infection upon effective contact with an infected vector. The latent or exposed class $E_{h}(t)$ (infected but not infectious), LF infected $I_{h}(t)$ (assumed infectious) and the recovered compartment $R(t)$. The vector population at time $t, N_{v}(t)$, on the other comprises the susceptible female mosquitoes $S_{v}(t)$. The exposed vector $E_{v}(t)$ also infected mosquitoes but not yet infectious and infected vector $I_{v}(t)$ with the potential pass on LF parasite through effective contact with susceptible humans. We assume homogeneous mixing of the population so that transmission occurs horizontally through standard incidence. The disease transmission vertically is ignored. The rate of infection of susceptible individuals is $\lambda_{h}$ and the rate at which the infected individuals infect the susceptible mosquito is $\lambda_{v}$. The susceptible humans $S_{h}$ are replenished through births and immigration at a constant rate $\Lambda$ and recovery of infected individuals at a constant rate of $\omega(\omega>0)$. The Susceptible human population $S_{h}$ is decreased by infection with LF through contact with infected mosquito at a rate $\lambda_{h}$ and move to exposed class. Exposed compartment gains from infection of susceptibles at a rate $\lambda_{h}$ and decreasing through chemoprophylaxis at a constant rate and progress to recovery class $R$ at a constant rate of $\delta$. The compartment is further decreased by the development to active LF $I_{h}$ at a constant rate $v$ and progress to the recovery state $R$ due to successfully treatment at the rate $\mathcal{E}$. Since the disease has temporary immunity, a fraction $\omega$ of individuals moves from the recovery state to susceptible state. It is assumed that $\lambda_{h}=\frac{\beta_{v h} \alpha I_{v}}{N_{v}}$ and $\lambda_{v}=\frac{\beta_{h v} \alpha I_{h}}{N_{h}}$ as in Tumwiine [22]. The term $\frac{\beta_{v h} \alpha I_{v}}{N_{v}}$ denotes the rate at which the human hosts $S_{h}$ get infected by infected mosquitoes $I_{v}$ and $\frac{\beta_{h v} \alpha I_{h}}{N_{h}}$ refers to the rate at which the susceptible mosquitoes $S_{v}$ get infected by the infected human hosts. It indicates that the rate of infection of susceptible human $S_{h}$ by infected mosquito $I_{v}$ is dependent on proportion of available infected vector on the total number of vector population. $\beta_{v h}$ is the probability that a human host becomes infectious and $\beta_{h v}$ is the probability that susceptible mosquitoes become infected by biting infected human while $\alpha$ refers to mosquito biting rate. Therefore the total human population is given by

$$
N_{h}=S_{h}+E_{h}+I_{h}+R
$$

and the total vector (mosquito) population is

$$
N_{v}=S_{v}+E_{v}+I_{v}
$$

The proposed model satisfies the assumption that host and vector populations are not constant, there is no vertical transmission meaning that all newly born are susceptible to infection and we assume that recovered hosts have temporary immunity that can be lost and be susceptible to infection again, but there is no recovery from infection to vector class.

Taking into account the above considerations and assumptions, we then have the following schematic flow diagram: 


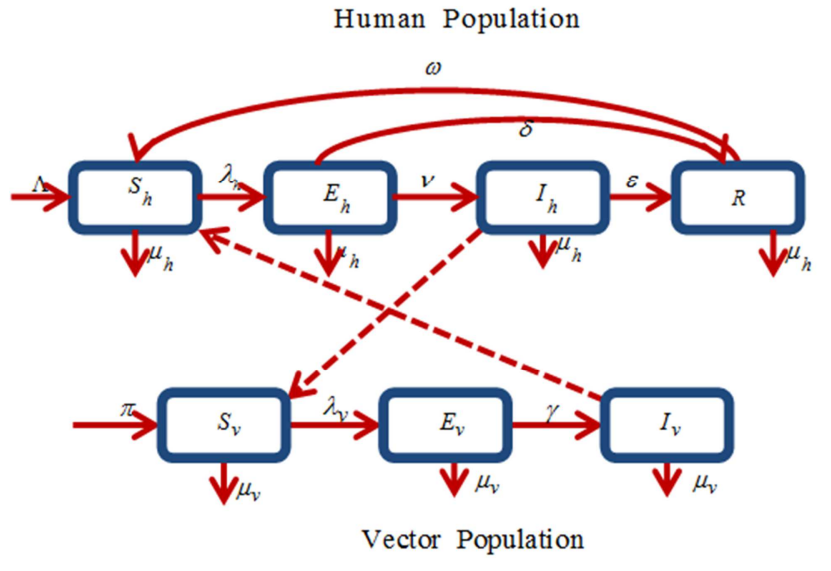

Figure 1. The Lymphatic Filariasis Model.

The model is thus governed by the following system of non-linear ordinary differential equations:

$$
\begin{aligned}
& \frac{d S_{h}}{d t}=\Lambda-\lambda_{h} S_{h}+\omega R-\mu_{h} S_{h} \\
& \frac{d E_{h}}{d t}=\lambda_{h} S_{h}-\left(v+\delta+\mu_{h}\right) E_{h} \\
& \frac{d I_{h}}{d t}=v E_{h}-\left(\mu_{h}+\varepsilon\right) I_{h} \\
& \frac{d R}{d t}=\delta E_{h}+\varepsilon I_{h}-\left(\omega+\mu_{h}\right) R \\
& \frac{d S_{v}}{d t}=\pi-\lambda_{v} S_{v}-\mu_{v} S_{v} \\
& \frac{d E_{v}}{d t}=\lambda_{v} S_{v}-\left(\gamma+\mu_{v}\right) E_{v} \\
& \frac{d I_{v}}{d t}=\gamma E_{v}-\mu_{v} I_{v}
\end{aligned}
$$

with non-negative initial conditions and $N_{h}(0)>0$, $N_{v}(0)>0$. The terms $\lambda_{h}$ and $\lambda_{v}$ are as defined above.

\section{Model Analysis}

\subsection{Positivity of Solutions}

For the model $(3-9)$ to be epidemiologically meaningful and well posed, we need to prove that all state variables are non-negative $\forall t \geq 0$.

Lemmal.

Let $\left\{\begin{array}{c}\left(S_{h}(0), S_{v}(0)\right)>0, \\ \left(E_{h}(0), I_{h}(0), R(0), E_{v}(0), I_{v}(0) \geq 0\right)\end{array}\right\} \in \Gamma$

Then the solution $\left\{S_{h}, E_{h}, I_{h}, R, S_{v}, E_{v}, I_{v}\right\}(t)$ of the model system $(3-9)$ is positive for all $t \geq 0$.
Proof. From the first equation of system (3), we have

$$
\frac{d S_{h}}{d t}=\Lambda-\lambda_{h} S_{h}+\omega R-\mu_{h} S_{h} \geq-\left(\lambda_{h}+\mu_{h}\right) S_{h}
$$

which upon integration gives

$$
S_{h}(t) \geq S_{h}(0) e^{-\left(\int \lambda_{h}+\mu_{h}\right) d t} \geq 0 \text { as } t>\infty,
$$

we obtain $S_{h}(t) \geq S_{h}(0)$

Similarly, it can be shown that all the remaining state variables of the system $(3-9)$ are also positive for all $t>0$. Hence, all feasible solution of system (3-9) enters the invariant region

$$
\Gamma=\left\{S_{h}, E_{h}, I_{h}, R, S_{v}, E_{v}, I_{v}\right\} .
$$

\subsection{Steady State Solutions}

Setting the right and side of system $(3-9)$ to zero in terms of $\lambda_{h}^{*}$ and $\lambda_{v}^{*}$ we obtain the following

$$
\begin{gathered}
S_{h}^{*}=\frac{\Lambda}{\mu_{h}+(1-\omega Q) \lambda_{h}^{*}} \\
E_{h}^{*}=\left(\frac{1}{v+\delta+\mu_{h}}\right) \lambda_{h}^{*} S_{h}^{*} \\
I_{h}^{*}=\left(\frac{1}{\left(\varepsilon+\mu_{h}\right)}\right)\left(\frac{v}{v+\delta+\mu_{h}}\right) \lambda_{h}^{*} S_{h}^{*} \\
R^{*}=Q \lambda_{h}^{*} S_{h}^{*} \\
S_{v}^{*}=\frac{\pi}{\left(\mu_{v}+\lambda_{v}\right)} \\
E_{v}^{*}=\left(\frac{1}{\gamma+\mu_{v}}\right) \lambda_{v}^{*} S_{v}^{*} \\
I_{v}^{*}=\left(\frac{\gamma}{\mu_{v}\left(\gamma+\mu_{v}\right)}\right) \lambda_{v}^{*} S_{v}^{*}
\end{gathered}
$$

where

$$
Q=\left(\frac{1}{\left(\omega+\mu_{h}\right)}\right)\left[\frac{\delta}{v+\delta+\mu_{h}}+\left(\frac{\varepsilon}{\left(\varepsilon+\mu_{h}\right)}\right)\left(\frac{v}{v+\delta+\mu_{h}}\right)\right] \leq 1
$$

Substituting $I_{v}^{*}$ and $I_{h}^{*}$ in the expression for the forces of infection $\lambda_{v}^{*}$ and $\lambda_{h}^{*}$ respectively, we obtain

$$
\lambda_{v}^{*}=\frac{\beta_{h v} \alpha v \lambda_{h}^{*} S_{h}^{*}}{\left(v+\delta+\mu_{h}\right)\left(\varepsilon+\mu_{h}\right) N_{h}}
$$




$$
\lambda_{h}^{*}=\frac{\beta_{v h} \alpha \gamma \lambda_{v}^{*} S_{v}^{*}}{\left(\gamma+\mu_{v}\right) \mu_{v} N_{v}}
$$

Upon substitution of $\lambda_{h}^{*}$ into $\lambda_{v}^{*}$ we obtain

$$
\begin{gathered}
\lambda_{v}^{*}=\left(\frac{\alpha \beta_{h v} v}{\left(v+\delta+\mu_{h}\right)\left(\varepsilon+\mu_{h}\right)}\right)\left(\frac{\alpha \beta_{v h} \gamma}{\left(\gamma+\mu_{v}\right) \mu_{v}}\right) \frac{S_{h}^{*}}{N_{h}} \frac{S_{v}^{*}}{N_{v}} \lambda_{v}^{*} \\
\lambda_{v}^{*}=0 \\
\text { or } R_{e}^{2} S_{v}^{*} S_{h}^{*}=N_{v}^{*} N_{h}^{*}
\end{gathered}
$$

where $R_{e}^{2}=\left(\frac{\alpha}{\mu_{v}}\right)\left(\frac{\gamma}{\gamma+\mu_{v}}\right) \beta_{h v}\left(\frac{\alpha}{\varepsilon+\mu_{h}}\right)\left(\frac{v}{v+\delta+\mu_{h}}\right) \beta_{v h}$ we also observe that

$$
N_{v}^{*}=S_{v}^{*}+E_{v}^{*}+I_{v}^{*}=S_{v}^{*}+Q_{v} \lambda_{v}^{*} S_{v}^{*}
$$

and $N_{h}^{*}=S_{h}^{*}+E_{h}^{*}+I_{h}^{*}+R^{*}=S_{h}^{*}+Q_{h} \lambda_{h}^{*} S_{h}^{*}$

with $Q_{v}=\frac{1}{\left(\gamma+\mu_{v}\right)}+\frac{\gamma}{\mu_{v}\left(\gamma+\mu_{v}\right)}=\frac{1}{\mu_{v}}$

and $Q_{h}=\frac{1}{\left(v+\delta+\mu_{h}\right)}+\frac{\gamma}{\mu_{v}\left(\gamma+\mu_{v}\right)}+Q$

Substituting for $N_{v}^{*}$ and $N_{h}^{*}$ in equation (11) above we have

$$
A \lambda_{v}^{2}+B \lambda_{v}+C=0
$$

where

$$
\begin{gathered}
A=\left(Q_{v}^{2}+Q_{v} Q_{h} R_{e_{v}}\right) \\
B=Q_{v}\left(\sqrt{2}-R_{e}\right)\left(\sqrt{2}+R_{e}\right)+Q_{h} R_{e_{v}} \\
C=\left(1-R_{e}\right)\left(1+R_{e}\right)
\end{gathered}
$$

\subsection{Disease-Free Equilibrium Point}

The solution $\lambda_{v}^{*}=0$ of (10) lead to the disease free equilibrium point given by $E_{0}=\left(\frac{\Lambda}{\mu_{h}}, 0,0,0, \frac{\pi}{\mu_{v}}, 0,0\right)$

\subsection{Existence of Endemic Equilibrium Point $E_{1}$}

The solution $A \lambda_{v}^{2}+B \lambda_{v}+C=0$ of (11) leads to the endemic equilibrium point given by

$$
S_{h}^{*}=\frac{\Lambda}{\mu_{h}+(1-\omega Q) \lambda_{h}^{*}}
$$

$$
\begin{gathered}
E_{h}^{*}=\left(\frac{1}{v+\delta+\mu_{h}}\right) \lambda_{h}^{*} S_{h}^{*} \\
I_{h}^{*}=\left(\frac{1}{\left(\varepsilon+\mu_{h}\right)}\right)\left(\frac{v}{v+\delta+\mu_{h}}\right) \lambda_{h}^{*} S_{h}^{*} \\
R^{*}=Q \lambda_{h}^{*} S_{h}^{*} \\
S_{v}^{*}=\frac{\pi}{\left(\mu_{v}+\lambda_{v}\right)} \\
E_{v}^{*}=\left(\frac{1}{\gamma+\mu_{v}}\right) \lambda_{v}^{*} S_{v}^{*} \\
I_{v}^{*}=\left(\frac{\gamma}{\mu_{v}\left(\gamma+\mu_{v}\right)}\right) \lambda_{v}^{*} S_{v}^{*}
\end{gathered}
$$

Where

$$
Q=\left(\frac{1}{\left(\omega+\mu_{h}\right)}\right)\left[\frac{\delta}{v+\delta+\mu_{h}}+\left(\frac{\varepsilon}{\left(\varepsilon+\mu_{h}\right)}\right)\left(\frac{v}{v+\delta+\mu_{h}}\right)\right] \leq 1
$$

\subsection{Reproduction Number $R_{e}$}

The basic reproduction number or contact number $R_{e}$ represents the average number of secondary infections that a single infection host can generate in a totally susceptible population of hosts and vectors. We calculate the basic reproduction number $R_{e}$ by using the next generation method on the system $(3-9) . R_{e}$ is obtained by taking the largest (dominant) eigenvalue (spectral radius) of

$$
A=\left[\frac{\partial F_{i}\left(E_{0}\right)}{\partial x_{i}}\right] \cdot\left[\frac{\partial V_{i}\left(E_{0}\right)}{\partial x_{i}}\right]^{-1}=F V^{-1}
$$

where $F_{i}$ is the rate of appearance of new infections and $V_{i}=V_{i}^{-}-V_{i}^{+}$is the is the net rate of transfer of into compartment $\mathrm{i}$; with $V_{i}^{-}$denoting transfer out of compartment $i$ and $V_{i}^{+}$the transfer of individuals into compartment $i$. This model has four (4) infected classes, thus $m=4$; and are ordered as follows: $E_{h}, I_{h}, E_{v}$, and $I_{v}$. The matrices $F$ and $V$ are obtained from model $(3-9)$ as

$$
F=\left[\begin{array}{llll}
\frac{\partial f_{1}\left(E_{0}\right)}{\partial E_{h}} & \frac{\partial f_{1}\left(E_{0}\right)}{\partial I_{h}} & \frac{\partial f_{1}\left(E_{0}\right)}{\partial E_{v}} & \frac{\partial f_{1}\left(E_{0}\right)}{\partial I_{v}} \\
\frac{\partial f_{2}\left(E_{0}\right)}{\partial E_{h}} & \frac{\partial f_{2}\left(E_{0}\right)}{\partial I_{h}} & \frac{\partial f_{2}\left(E_{0}\right)}{\partial E_{v}} & \frac{\partial f_{2}\left(E_{0}\right)}{\partial E_{v}} \\
\frac{\partial f_{3}\left(E_{0}\right)}{\partial E_{h}} & \frac{\partial f_{3}\left(E_{0}\right)}{\partial I_{h}} & \frac{\partial f_{3}\left(E_{0}\right)}{\partial E_{v}} & \frac{\partial f_{3}\left(E_{0}\right)}{\partial I_{v}} \\
\frac{\partial f_{4}\left(E_{0}\right)}{\partial E_{h}} & \frac{\partial f_{4}\left(E_{0}\right)}{\partial I_{h}} & \frac{\partial f_{4}\left(E_{0}\right)}{\partial E_{v}} & \frac{\partial f_{4}\left(E_{0}\right)}{\partial I_{v}}
\end{array}\right]
$$




$$
\mathbf{F}=\left[\begin{array}{cccc}
0 & 0 & 0 & \frac{\beta_{v h} \alpha \Lambda \mu_{v}}{\pi \mu_{h}} \\
0 & 0 & 0 & 0 \\
0 & \frac{\beta_{h v} \alpha \pi \mu_{h}}{\Lambda \mu_{v}} & 0 & 0 \\
0 & 0 & 0 & 0
\end{array}\right]
$$

The Jacobian matrix of $\mathbf{V}$ evaluated at $E_{0}$ is given by

$$
\mathbf{V}=\left[\begin{array}{cccc}
\left(v+\delta+\mu_{h}\right) & 0 & 0 & 0 \\
-v & \left(\varepsilon+\mu_{h}\right) & 0 & 0 \\
0 & 0 & \left(\gamma+\mu_{v}\right) & 0 \\
0 & 0 & -\gamma & \mu_{v}
\end{array}\right]
$$

$R_{e}$ is defined as the spectral radius (dominant eigenvalues) of the matrix $\mathbf{F V}^{-1}$ which is

$$
R_{e}=\sqrt{\frac{\beta_{v h} \beta_{h v} \alpha^{2} v \gamma}{\left(v+\delta+\mu_{h}\right)\left(\varepsilon+\mu_{h}\right)\left(\gamma+\mu_{v}\right) \mu_{v}}}
$$

$$
J_{E_{0}}=\left[\begin{array}{ccccccc}
-\mu_{h} & 0 & 0 & \omega & 0 & 0 & -\alpha \beta_{v h} \\
0 & -\left(v+\delta+\mu_{h}\right) & 0 & 0 & 0 & 0 & \alpha \beta_{v h} \\
0 & v & -\left(\varepsilon+\mu_{h}\right) & 0 & 0 & 0 & 0 \\
0 & \delta & \varepsilon & -\left(\omega+\mu_{h}\right) & 0 & 0 & 0 \\
0 & 0 & -\alpha \beta_{h v} & 0 & -\mu_{v} & 0 & 0 \\
0 & 0 & \alpha \beta_{h v} & 0 & 0 & -\left(\gamma+\mu_{v}\right) & 0 \\
0 & 0 & 0 & 0 & 0 & \gamma & -\mu_{v}
\end{array}\right]
$$

The characteristic polynomial of $J_{E_{0}}$ is given by $\left|J_{E_{0}}-\lambda I\right|=0$ giving rise to $\lambda_{1}=-\mu_{h}$ and the eigenvalues

$$
\lambda_{2,3}=\left|\begin{array}{cc}
-\left(v+\delta+\mu_{h}+\lambda\right) & 0 \\
v & -\left(\varepsilon+\mu_{h}+\lambda\right)
\end{array}\right|=0
$$

which yields $\lambda_{2}=-\left(v+\delta+\mu_{h}\right)$ and $\lambda_{3}=-\left(\varepsilon+\mu_{h}\right)$, $\lambda_{4}=-\left(\omega+\mu_{h}\right), \quad \lambda_{5}=-\mu_{v} \quad$ again eigenvalues $\lambda_{6,7}=\left|\begin{array}{cc}-\left(\gamma+\mu_{v}+\lambda\right) & 0 \\ \gamma & -\mu_{v}-\lambda\end{array}\right|=0$ which gives $\lambda_{6}=-\mu_{v}$ and $\lambda_{7}=-\left(\gamma+\mu_{v}\right)$, Since $\lambda_{1}<\lambda_{2}<\ldots<\lambda_{7}<0$ (both eigenvalues are real and negative) then the equilibrium is asymptotically stable.

\subsection{Global Stability of the Disease Free Equilibrium Point} $E_{0}$

Theorem 2: The Disease Free Equilibrium point $E_{0}$ is asymptotically stable if $R_{e}<1$ and unstable if $R_{e}>1$

Proof: We use the comparison theorem to prove theorem 2 as in Lakshmikantham [13], Niger [16]. We consider the infected class components where their rates of change can be

$$
R_{e}=\sqrt{R_{e v} R_{e h}}
$$

The basic reproduction number $R_{e}$ is used to determine whether the disease becomes persistent or dies out depends on magnitude of $R_{e}$. Stability of the equilibrium points can be analyzed using $R_{e}$.

\subsection{Local Stability of Disease Free Equilibrium Point}

Theorem 1: The disease free equilibrium point is locally

Asymptotically stable if and only if $R_{e}<1$, and is unstable if $R_{e}>1$.

Proof: The disease-free steady state, $E_{0}$ is given by $\left(\frac{\Lambda}{\mu_{h}}, 0,0,0, \frac{\pi}{\mu_{v}}, 0,0\right)$.

The Jacobian matrix of the system $(3-9)$ evaluated at $E_{0}$ is given by

written as

$$
\left[\begin{array}{c}
E_{h}^{\prime}(t) \\
I_{h}^{\prime}(t) \\
E_{v}^{\prime}(t) \\
I_{v}^{\prime}(t)
\end{array}\right]=(\mathbf{F}-\mathbf{V})\left[\begin{array}{c}
E_{h} \\
I_{h} \\
E_{v} \\
I_{v}
\end{array}\right]-\left[\begin{array}{c}
\beta_{v h} \alpha I_{v}\left(\frac{\Lambda \mu_{v}}{\pi \mu_{h}}-\frac{S_{h}}{N_{v}}\right) \\
0 \\
\beta_{h v} \alpha I_{h}\left(\frac{\pi \mu_{h}}{\Lambda \mu_{v}}-\frac{S_{v}}{N_{h}}\right) \\
0
\end{array}\right]
$$

where the matrices $\mathbf{F}$ and $\mathbf{V}$ are given by (14) and (15). However, considering that $S_{h} \leq \frac{\Lambda \mu_{v}}{\pi \mu_{h}} \quad$, then $\frac{S_{h}}{N_{v}} \leq \frac{\Lambda \mu_{v}}{\pi \mu_{h}} \quad \forall t \geq 0 \quad$ in $\quad$ the region $\quad \Gamma \quad$. Similarly, $\frac{S_{v}}{N_{h}} \leq \frac{\pi \mu_{h}}{\Lambda \mu_{v}} \quad \forall t \geq 0$ in the region $\Gamma$. Hence we have

$$
\left[\begin{array}{c}
E_{h}^{\prime}(t) \\
I_{h}^{\prime}(t) \\
E_{v}^{\prime}(t) \\
I_{v}^{\prime}(t)
\end{array}\right] \leq(\mathbf{F}-\mathbf{V})\left[\begin{array}{c}
E_{h} \\
I_{h} \\
E_{v} \\
I_{v}
\end{array}\right]
$$


where

$$
(\mathbf{F}-\mathbf{V})=\left[\begin{array}{cccc}
-\left(v+\delta+\mu_{h}\right) & 0 & 0 & \frac{\beta_{v h} \alpha \Lambda \mu_{v}}{\pi \mu_{h}} \\
v & -\left(\varepsilon+\mu_{h}\right) & 0 & 0 \\
0 & \frac{\beta_{h v} \alpha \pi \mu_{h}}{\Lambda \mu_{v}} & -\left(\gamma+\mu_{v}\right) & 0 \\
0 & 0 & \gamma & -\mu_{v}
\end{array}\right]
$$

Moreover, having all eigenvalues of the matrix $\mathbf{F}-\mathbf{V}$ with negative real parts, it follows that the differential inequality system (18) is stable for $R_{e}<1$ Niger [14]. Consequently $\left(E_{h}, I_{h}, E_{v}, I_{v}\right) \rightarrow(0,0,0,0)$ as $t \rightarrow \infty$.

Thus, by comparison theorem Lakshmikantham [13] $\left(E_{h}, I_{h}, E_{v}, I_{v}\right) \rightarrow(0,0,0,0)$ as $t \rightarrow \infty$. Evaluating system $(3-9) \quad$ at $\quad E_{h}=I_{h}=E_{v}=I_{v}=0 \quad$ gives $\quad S_{h} \rightarrow \frac{\Lambda}{\mu_{h}} \quad$ and $S_{v} \rightarrow \frac{\pi}{\mu_{v}}$ for $R_{e}<1$. Hence the Disease Free Equilibrium point $E_{0}$ is globally asymptotically stable for $R_{e}<1$.

\subsection{Stability of the Endemic Equilibrium Point $E_{1}$}

\subsubsection{Non-existence of Endemic Equilibria for $R_{e}<1$}

Models that exhibit backward bifurcation are characterized by the presence of two equilibria.

When the associated $R_{e}<1$. This property is now investigated. From equation (12)

It can be noted from equation (12) that the coefficient $\mathrm{A}$ is always positive.

Case i: When $R_{e}<1$ then $B>0$ and $C>0$. Thus by using Descartes' rule of sign and Routh Hurwitz criterion, the quadratic (12) has no positive roots. Therefore system (3-9) has no endemic steady states when $R_{e}<1$.

Case ii. When $R_{e}=1, B>0$ and $C=0$, the quadratic (12) reduces to $\lambda_{v}=-\frac{B}{A}<0$.

Case iii. When $R_{e}>1$, always $C<0$ and there are two possibilities of the values of $\mathrm{B}$, either $B>0$ or $B<0$.

In the case, when $B>0$ the quadratic (12) has only one real positive root likewise when $B<0$ the quadratic (12) has only one real positive root.

Theorem 3: The Lymphatic Filariasis model $(3-9)$ has at least one endemic equilibrium $E_{1}$ whenever $R_{e}>1$. If $R_{e}<1$, the model (3-9) has no endemic equilibria.

Theorem 3, shows that the model exhibits forward bifurcation, due to none endemic equilibria whenever $R_{e}<1$. For detailed exposition of forward bifurcation, the stability of endemic equilibrium is again determined through the investigation of the possibility of existence of the forward bifurcation using Center Manifold theorem. According to Chavez [5] the result indicates the possibility of forward bifurcation. This is demonstrated graphically in Fig. 2 (the figure shows a forward bifurcation).

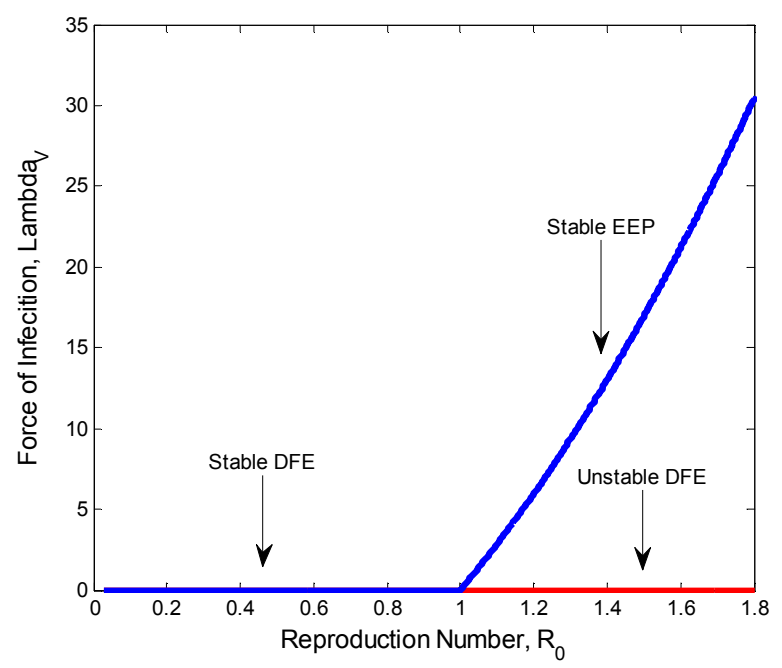

Figure 2. Forward Bifurcation.

Figure 2 illustrates a forward bifurcation of the force of infection at equilibrium against the reproduction number $R_{e}$ of the model $(3-9)$. The stationary solutions occur at $R_{e}=1$. If $R_{e}<1$ no biologically meaning for endemic stationary solution exist since the disease-free stationary solution is a global attractor, but if $R_{e}>1$ the endemic solution exists and is a global attractor. This is referred to as a forward bifurcation because in the neighbourhood of the bifurcation point, the endemic disease prevalence is an increasing function of $R_{0}$.

\subsubsection{Global Stability EEP}

In this section, we investigate the global stability of the EEP using Lyapunov function technique developed and explored by Korobeinikov [10, 11, 12]. We construct Lyapunov function of the form,

$$
L=\sum a_{i}\left(X_{i}-X_{i}^{*} \ln X_{i}\right)
$$

where $a_{i}$ is properly selected constants, $X_{i}$ is the population of the $i^{\text {th }}$ compartment, and $X_{i}^{*}$ is the equilibrium point. The approach has been found to be useful for compartmental epidemic models with any number of compartments Korobeinikov $[8,9,10]$. Thus, we consider the Lyapunov function of the form

$$
\begin{aligned}
L & =a_{1}\left(S_{h}-S_{h}^{*} \ln S_{h}\right)+a_{2}\left(E_{h}-E_{h}^{*} \ln E_{h}\right)+a_{3}\left(I_{h}-I_{h}^{*} \ln E_{h}\right) \\
& +a_{4}\left(R-R^{*} \ln R\right)+a_{5}\left(S_{v}-S_{v}^{*} \ln S_{v}\right)+a_{6}\left(E_{v}-E_{v}^{*} \ln E_{v}\right) \\
& +a_{7}\left(I_{v}-I_{v}^{*} \ln I_{v}\right)
\end{aligned}
$$


where, $a_{1}, a_{2}, a_{3}, \ldots, a_{7}$ are positive constants. Differentiating our Lyapunov function with respect to time we have,

$$
\begin{aligned}
\frac{d L}{d t} & =a_{1}\left(1-\frac{S_{h}^{*}}{S_{h}}\right) \frac{d S_{h}}{d t}+a_{2}\left(1-\frac{E_{h}^{*}}{E_{h}}\right) \frac{d E_{h}}{d t}+a_{3}\left(1-\frac{I_{h}^{*}}{I_{h}}\right) \frac{d I_{h}}{d t} \\
& +a_{4}\left(1-\frac{R^{*}}{R}\right) \frac{d R}{d t}+a_{5}\left(1-\frac{S_{v}^{*}}{S_{v}}\right) \frac{d S_{v}}{d t}+a_{6}\left(1-\frac{E_{v}^{*}}{E_{v}}\right) \frac{d E_{v}}{d t} \\
& +a_{7}\left(1-\frac{I_{v}^{*}}{I_{v}}\right) \frac{d I_{v}}{d t}
\end{aligned}
$$

From the system $(3-9)$ we have,

$$
\begin{aligned}
& \frac{d L}{d t}=-a_{1}\left(1-\frac{S_{h}^{*}}{S_{h}}\right)^{2} \mu_{h} S_{h}-a_{5}\left(1-\frac{S_{v}^{*}}{S_{v}}\right)^{2} \mu_{v} S_{v} \\
& -a_{1}\left(1-\frac{S_{h}^{*}}{S_{h}}\right)\left(1-\frac{I_{v}^{*} S_{h}^{*}}{I_{v} S_{h}}\right) \frac{\beta_{v h} \alpha \mu_{v} I_{v} S_{h}}{\pi} \\
& +a_{1}\left(1-\frac{S_{h}^{*}}{S_{h}}\right)\left(1-\frac{R^{*}}{R}\right) \omega R \\
& +a_{2}\left(1-\frac{E_{h}^{*}}{E_{h}}\right)\left(1-\frac{I_{v}^{*} S_{h}^{*} E_{h}}{I_{v} S_{h} E_{h}^{*}}\right) \frac{\alpha \beta_{v h} \mu_{v} I_{v} S_{h}}{\pi} \\
& +a_{3}\left(1-\frac{I_{h}^{*}}{I_{h}}\right)\left(1-\frac{E_{h}^{*} I_{h}}{E_{h} I_{h}^{*}}\right) v E_{h} \\
& -a_{4}\left(1-\frac{R^{*}}{R}\right)\left(1-\frac{E_{h}^{*} R}{E_{h} R^{*}}\right) \delta E_{h} \\
& +a_{4}\left(1-\frac{R^{*}}{R}\right)\left(1-\frac{I_{h}^{*} R}{I_{h} R^{*}}\right) \varepsilon I_{h} \\
& -a_{5}\left(1-\frac{S_{v}^{*}}{S_{v}}\right)\left(1-\frac{I_{h}^{*} S_{v}^{*}}{I_{h} S_{v}}\right) \frac{\alpha \beta_{h v} \mu_{h} I_{h} S_{v}}{\Lambda} \\
& +a_{6}\left(1-\frac{E_{v}^{*}}{E_{v}}\right)\left(1-\frac{I_{h}^{*} S_{v}^{*} E_{v}}{I_{h} S_{v} E_{v}^{*}}\right) \frac{\alpha \beta_{h v} \mu_{h} I_{h} S_{v}}{\Lambda} \\
& +a_{7}\left(1-\frac{I_{v}^{*}}{I_{v}}\right)\left(1-\frac{E_{v}^{*} I_{v}}{E_{v} I_{v}^{*}}\right) \gamma E_{v}
\end{aligned}
$$

This this can be written as:

$$
\frac{d L}{d t}=-a_{1}\left(1-\frac{S_{h}^{*}}{S_{h}}\right)^{2} \mu_{h} S_{h}-a_{6}\left(1-\frac{S_{v}^{*}}{S_{v}}\right)^{2} \mu_{v} S_{v}+F(\Re)
$$

where, $F(\Re)$ is the balance of the right hand terms of $(24)$.

Following the approach of Bowong [3], Korobeinikov [10] and McCluskey [14] $F$ is non-positive for
$S_{h}, E_{h}, I_{h}, R, S_{v}, E_{v}, I_{v}>0$ and therefore,

$$
\begin{gathered}
\frac{d L}{d t}=0 \text { if } \\
S_{h}=S_{h}^{*}, E_{h}=E_{h}^{*}, I_{h}=I_{h}^{*}, R=R^{*}, S_{v}=S_{v}^{*}, E_{v}=E_{v}^{*}, I_{v}=I_{v}^{*} \\
\frac{d L}{d t}<0 \text { for? } S_{h} E_{h}, I_{h} R S_{v} E_{v} I_{v}>.
\end{gathered}
$$

Thus, if $R_{\text {eff }}>1$ then, model system (3-9) has endemic equilibrium point $E_{1}$ which is globally asymptotically stable.

\section{Sensitivity Analysis}

In general the results of the model depends on the model structure (compartments and flows) and model parameter values. Changes in model parameters may result in incorrect disease transmission or assessment of impact of infection control systems. Numerical sensitivity analysis of the basic reproduction number against model parameters based on the technique developed by Chitnis [6] is presented. The aim of the study is explore model output sensitivity with changes in model parameters or identify parameters with important uncertainties on the model output. The process involves the determination of sensitivity indices that measure the relative change of state variables with changes in parameter values. We compute the sensitivity indices using an analytical expression for the sensitivity of $R_{e}$ as $r_{q}^{R_{e}}=\frac{\partial R_{e}}{\partial q} \times \frac{q}{R_{e}}$, to each parameter involved in $R_{e}$. For example sensitivity index of $R_{e}$ with respect to $\alpha$ and $\gamma$ are

$$
r_{\alpha}^{R_{e}}=\frac{\partial R_{e}}{\partial \alpha} \times \frac{\alpha}{R_{e}}=9.7912, \quad r_{\gamma}^{R_{e}}=\frac{\partial R_{e}}{\partial \gamma} \times \frac{\gamma}{R_{e}}=0.01923
$$

respectively. Other indices include $r_{\beta_{v h}}^{R_{e}}, r_{\beta_{h v}}^{R_{e}}, r_{\beta_{\delta}}^{R_{e}}, r_{\mu_{v}}^{R_{e}}$, $r_{\mu_{h}}^{R_{e}}, r_{v}^{R_{e}}$ and $r_{\varepsilon}^{R_{e}}$ and are presented in table 1 below.

Table 1. Sensitivity Indices of $R_{e}$.

\begin{tabular}{llll}
\hline Parameter & Parameter Value & Sensitivity Index & Source \\
\hline$\alpha$ & 0.29 & +9.7912 & Ishikawa [7] \\
$\delta$ & 0.125 & -8.20209 & Assumed \\
$\mu_{v}$ & 0.03 & -0.51923 & Assumed \\
$\beta_{h v}$ & 0.083 & +0.5 & Miranda [15] \\
$\beta_{v h}$ & 0.86 & +0.49999 & Niger [16] \\
$\varepsilon$ & 0.00722 & -0.47375 & Niger [16] \\
$\nu$ & $1 / 17$ & +0.340347 & Blayneh [2] \\
$\mu_{h}$ & 0.0004 & -0.02733 & TACAIDS \\
$\gamma$ & 0.75 & +0.01923 & [20] \\
\hline
\end{tabular}


Table 1 show that the most sensitive parameter is the mosquito biting rate of mosquitoes $\alpha$ followed by treatment rate of exposed human individuals $\delta$. The death rate $\mu_{v}$ of mosquitoes is another important parameter in controlling the LF epidemic. This is followed by contact rate of infected host and susceptible vector $\beta_{h v}$ and the contact rate of infected vector with susceptible host $\beta_{v h}$. However, treatment rate $\mathcal{E}$ of infected human to recovery class is another important parameter followed by exposed human progression rate $v$ into infected human class. The interpretation of these results is that, on controlling the spread of Lymphatic Filariasis infections within the population the following should be taken into account; reduce mosquitoes biting rate, Increase in treatment rate among exposed individuals and increase mosquitoes mortality rate.

\section{Numerical Simulation}

In this section, we present numerical simulations of the model $(3-9)$ to predict the long term trends for both the host and vector population. This study used a set of parameter values from different sources from reviewed literature (Table 1). Thus, the dynamics of some epidemiological class of the model are simulated with time as well as the effects of sensitive parameters to illustrate the behaviour of the model.

Figure 3(a), shows that susceptible human population increases with time when the biting rate $\alpha$ of mosquitoes is reduced. On the other hand figure $3(\mathrm{~b})$, illustrate that when treatment rate $\delta$ of exposed individuals increases then susceptible population also increases, this is due to the fact that, increase in treatment of exposed individual increases the number of recovery human and consequently susceptible population increase. Moreover, increase in death rate $\mu_{v}$ of mosquito increases the susceptible population with time figure 3(c).
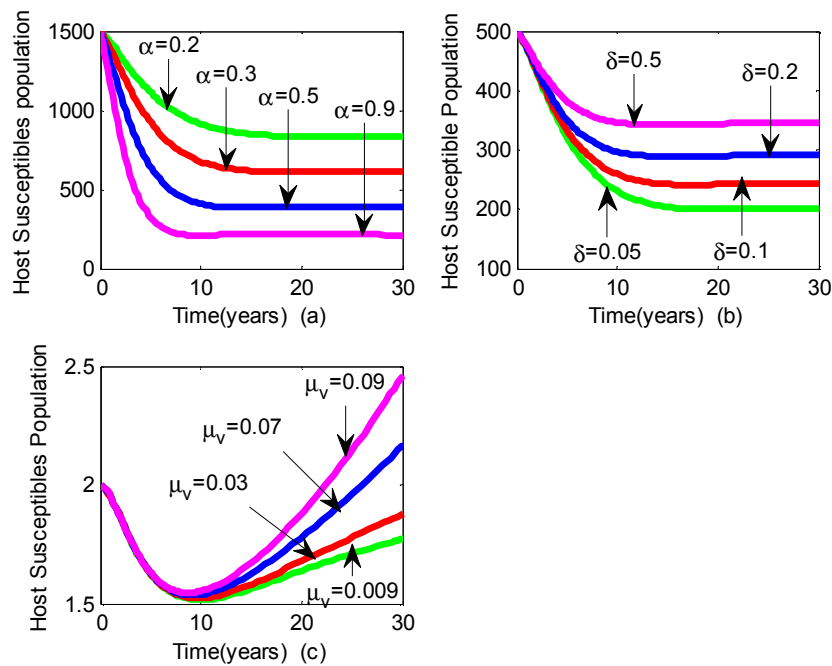

Figure 3. Variation of Human Susceptible Population for different values of $\alpha, \delta$ and $\mu_{v}$.
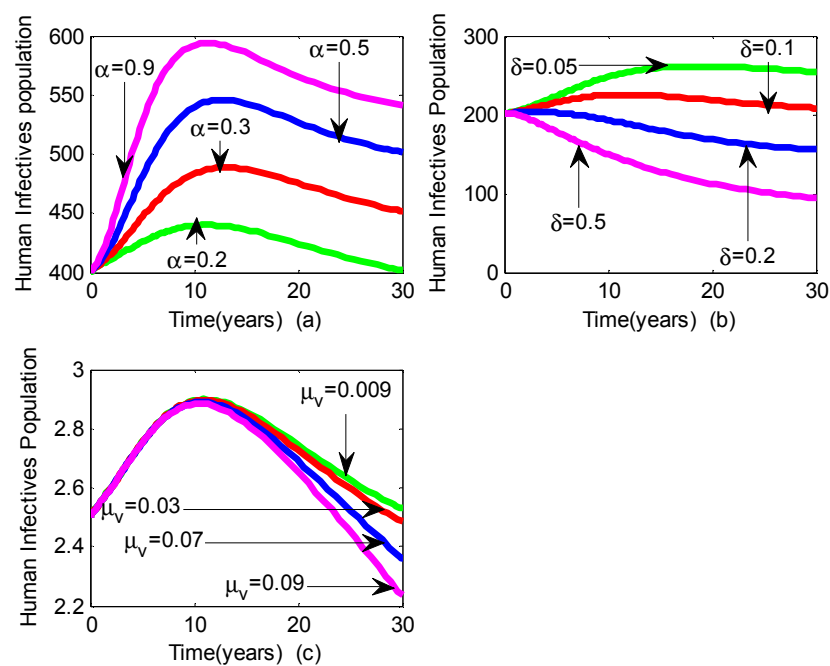

Figure 4. Variation of Human Infectives Population for different values of $\alpha, \delta$ and $\mu_{v}$.

We observe in figure 4(a), that the infected human population increases in the level of infection due to the increase of mosquito biting rate $\alpha$. Again, figure 4(b) shows that the infectious class reduces as treatment rate of the exposed class increase $\delta$. This implies that the introduced intervention to exposed class reduces the number of infected individuals who shift to infectious class. In figure 4(c), it is observed that the infected population reduces when there is an increase in mosquito mortality rate $\mu_{v}$. Therefore, we can conclude that, when mosquito biting rate is reduced among people, and mosquito death rate is increase this can reduce their level of lymphatic Filariasis infection as well as reducing the disease morbidity from the community.

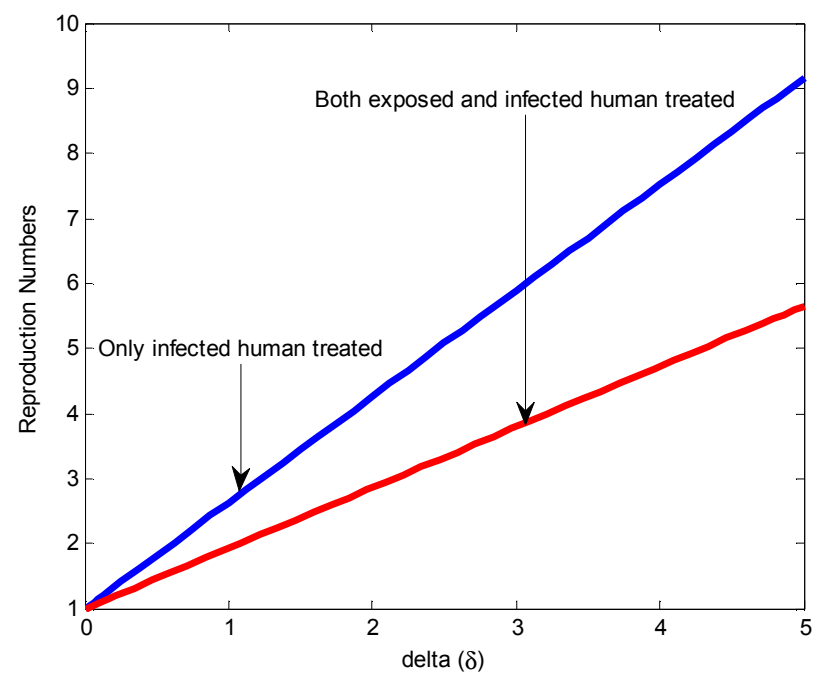

Figure 5. Variation of Reproduction numbers with different values of delta.

We ran the system to illustrate the effect of different parameter values on the reproduction numbers. These include the behaviour of the reproduction numbers when there is only treatment of infected individuals and when both exposed and infected individuals are involved in treatment. We found that 
reproduction number tend to decline when intervention is practiced to both populations.
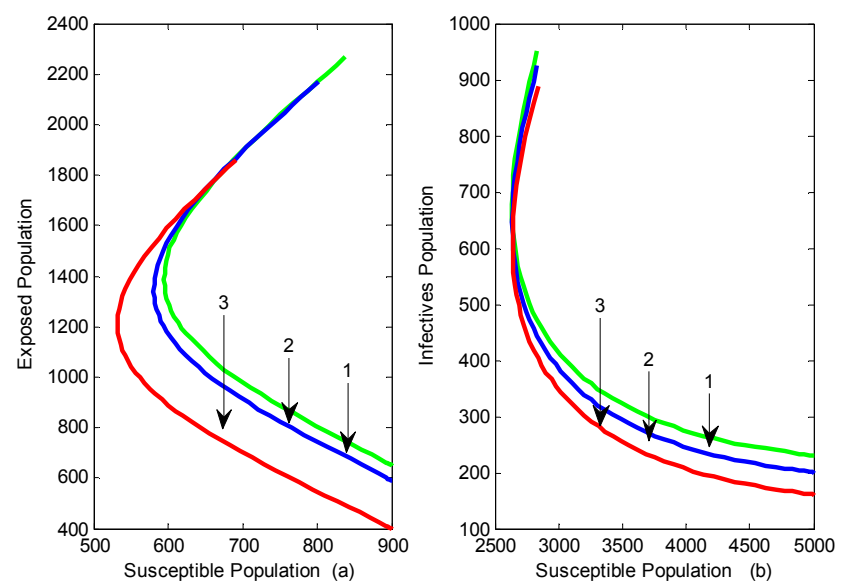

Figure 6. Phase plane for Exposed and Infected Human Individuals versus Susceptible Human Individuals.

Figure 6 (a)-(b) illussrate the phase portrait of the dynamics of susceptible human and exposed individuals as well as infected individuals population showing endemic equilibrium point for a given initial conditions as time increases. It can be observed that the trajectory for the initial populations end up in a situation where there is infected individuals, that is, the endemic equilibrium.

\section{Discussion and Conclusions}

A non-linear differential equation model has been constructed and analyzed to study the effect of chemoprophylaxis in the exposed individuals. Qualitative and numerical analysis of the model has been implemented. The Disease-free equilibrium and the Endemic equilibrium points were obtained and their stability analyzed. The study established that, treatment of exposed and symptomatic individuals have some effect on reducing the spread of lymphatic Filariasis infection.

Therefore, administration of chemoprophylaxis at early stage (asymptomatic infection), prevents the progression of LF infection to chronic infection and improve the quality of life in the community, but not to the levels necessary for disease elimination. This result suggests that effective control of lymphatic Filariasis may lie in treatment for both exposed and those displaying symptoms as well as control of vectors. Interventions targeting reduction in the biting rate of mosquitoes and increasing mortality rate of vectors in the population is critical in controlling the disease epidemic.

\section{References}

[1] C. Bhunu and S. Mushayabasa, "Transmission Dynamics of Lymphatic Filariasis, Mathematical Approach,” International Scholarly Research Network ISRN Biomathematics, vol 2012, Article ID 930130, 9 pages.
[2] K. Blayneh, Y. Cao, and H. Kwon, "Optimal Control of Vector borne Diseases: Treatment and Prevention," Discrete and Continuous Dynamical Systems Series B. 11(3) 2009. pp 1-xx.

[3] S. Bowong, J. J Tewa and J. C. Kamgang, "Transmission Dynamics of Tuberculosis Model," World Journal of Modelling and Simulation vol. 7, 2011, pp. 83-100.

[4] M. S. Chan, A. Srividya, R. A. Norman, S. P. Pani, K. D. Ramaiah, P. Vanamail, E. Michael, P. K. Das, and D. A. P. Bundy. "EPIFIL: A Dynamic Model of Infection and Disease in Lymphatic Filariasis," The American Society of Tropical Medicine and Hygiene 59(4), 1998, pp. 606-614.

[5] C. Chavez-Castillo and B. Song, "Dynamical Models of Tuberculosis and their Applications," Mathematical Biosciences and Engineering vol. 2, 2004 pp. 361-404.

[6] N. Chitnis, J. M. Hyman and J. M. Cushing, "Determining Important Parameters in the Spread of Malaria Through the Sensitivity Analysis of a Mathematical Model," Bulletin of Mathematical Biology vol. 70, 2008, pp. 1272-1296.

[7] H. A Ishikawa, A. Ishi, N. Nagai, H. Ohmae, H. Masakazu, S. Suguri and J. Leafa- sia. "A mathematical model for the transmission of Plasmodium vivax malaria," Parasitology International, vol. 52, 2003, pp. 81-93.

[8] P. Jambulingam, S. Subramanian, S. J de Vlas, C. Vinubala, W. A Stolk, "Mathematical modelling of lymphatic filariasis elimination programs in India: required duration of mass drug administration and post treatment level of infection indicators", Parasites and Vectors. November, 2015.

[9] R. J. Kastner, C. M. Stone, P. Steinmann, M. Tanner, and F. Tediosi, "What Is Needed to Eradicate Lymphatic Filariasis? A Model-Based Assessment on the Impact of Scaling Up Mass Drug Administration Programs" PLoS Negl. Trop Dis vol. 9 (10). 2015.

[10] A. Korobeinikov, "Global properties of basic virus dynamicals models,” Bull. Math. Biol. 2004, pp 879-883.

[11] Korobeinikov, Lyapunov functions and global proper ties for SEIR and SEIS epidemic models, Math. Med. Biol. vol 21 2004, pp. 75-83.

[12] A. Korobeinikov, Global properties of infectious dis ease models with nonlinear incidence, Bull. Math. Biol. Vol. 69, 2007, pp. 1871-1886.

[13] V. Lakshmikantham, S. Leela and A. A Martynyuk. "Stability analysis of nonlinear systems Pure and Ap plied Mathematics" Marcel Dekker, New York A Series of Monographs and Textbooks, Vol. 125. 2009. ISBN 0-8247-8067-1.

[14] C. C. McCluskey, "Lyapunov functions for tuberculo sis models with fast and slow Progression," Math Biosci Eng. 3, 2006, pp. 603-614.

[15] I. Miranda. Teboh-Ewungkem, N. Chandra, Podder, and B. A. Gumel. "Mathematical Study of the Role of Gametocytes and an Imperfect Vaccine on Malaria Transmission Dynamic," Bulletin of Mathematical Biology vol. 1007(11) 2009, pp. 538-009-9437-3.

[16] A. M, Niger and A. B. Gumel, "Mathematical Analy sis of the Role of Repeated Exposure on Malaria Transmission Dynamics," Differential Equations and Dynamical Systems, vol. 16 (3), 2008, pp 251-287. 
[17] W. A Stolk, S. J de Vlas, and J. D. F Habbema, “Advances and Challenges in Predicting the Impact of Lymphatic Filariasis Elimination Programmes," Fi larial Journal vol 5: 5, 2006.

[18] W. A. Stolk, Subramanian Swaminathan, Gerrit J. van Oortmarssen, P. K. Das, and J. Dik F. Habbe ma. "Prospects for Elimination of Bancroftian Filariasis by Mass Drug Treatment in Pondicherry, India” JID, 2003, 188.

[19] S. Swaminathan, Pani P Subash, Ravi Rengachari, Krishnamoorthy, Kaliannagounder and Das K Pradeep "Mathematical models for lymphatic filariasis transmission and control: Challenges and prospects," Parasites \& Vectors, 2008.
[20] TACAIDS-Tanzania, Joint Bi-annual HIV/AIDS Sector Review: Report of Technical Review: Dar-es-Salaam, Tanzania, 2008.

[21] J. Z. G. Tan, "The Elimination of Lymphatic Filariasis: A Strategy for Poverty Alleviation and Sustainable Development Perspectives from the Philippines", Filaria Journal, vol. 2. 2003.

[22] J. Tumwiine, J. Y. T. Mugisha and L. S. Luboobi. "On oscillatory pattern of malari dynamics in a population with temporary immunity," Computational and Mathematical Methods in Medicine vol. 8(3), 2007, pp. 191-203. 